\title{
Travelling wave solutions to the K-P-P equation: alternatives to Simon Harris' probabilistic analysis
}

\author{
A.E. Kyprianou \\ Department of Mathematics, Utrecht University, Budapestlaan 6, 3584CD Utrecht, The Netherlands
}

Received 16 February 2001; accepted 27 June 2003

\begin{abstract}
Recently Harris [Proc. Roy. Soc. Edinburgh Sect. A 129 (1999) 503], using probabilistic methods alone, has given new proofs for the existence, asymptotics and uniqueness of travelling wave solutions to the K-P-P equation. Following in this vein we outline alternative probabilistic proofs. Specifically the techniques are confined to the study of additive and multiplicative martingales and spinal path decompositions along the lines of [B. Chauvin, A. Rouault, Probab. Theory Related Fields 80 (1988) 299], [R. Lyons, in: K.B. Athreya, P. Jagers (eds.), Classical and Modern Branching Processes, Vol. 84, Springer-Verlag, New York, 1997, pp. 217-222] and [R. Lyons et al., Ann. Probab. 23 (1995) 1125]. We also make use of a new decomposition where the spine is a conditioned process. Some new results concerning martingale convergence are obtained as a by-product of the analysis.

(c) 2003 Elsevier SAS. All rights reserved.
\end{abstract}

\section{Résumé}

Harris a récemment donné [Proc. Roy. Soc. Edinburgh Sect. A 129 (1999) 503], par des méthodes purement probabilistes, de nouvelles preuves de l'existence, du comportement asymptotique et de l'unicité des propagations d'onde de l'équation KPP. En suivant la même veine, nous indiquons des preuves probabilistes alternatives. Les techniques sont limitées à l'étude de martingales additives et multiplicatives ainsi qu'aux décompositions «spinales» des trajectoires, employées dans [B. Chauvin, A. Rouault, Probab. Theory Related Fields 80 (1988) 299], [R. Lyons, dans : K.B. Athreya, P. Jagers (eds.), Classical and Modern Branching Processes, Vol. 84, Springer-Verlag, New York, 1997, pp. 217-222] et [R. Lyons et al., Ann. Probab. 23 (1995) 1125]. Nous utilisons également une nouvelle décomposition à partir d'un processus conditionné. De nouveaux résultats sur la convergence des martingales sont obtenus, chemin faisant. (c) 2003 Elsevier SAS. All rights reserved.

MSC: $60 \mathrm{~J} 80$

Keywords: Branching Brownian motion; K-P-P equation; Travelling wave solutions; Size biased measures; Bessel-3 processes; Brownian motion; Spine decompositions

E-mail address: kyprianou@math.uu.nl (A.E. Kyprianou).

0246-0203/\$ - see front matter C 2003 Elsevier SAS. All rights reserved.

doi:10.1016/j.anihpb.2003.06.001 


\section{Introduction}

A branching Brownian motion is constructed as follows. An initial ancestor begins its existence at the origin of one-dimensional Euclidean space. This individual moves according to an independent copy of standard Brownian motion $B=\{B(t): t \geqslant 0\}$ and produces a random number of offspring, $1+X$, after a time $\eta$ which is exponentially distributed with parameter $\beta>0$. We shall assume that $X$ has distribution $\left\{p_{k}: k \geqslant 0\right\}$ such that $m:=\sum_{k \geqslant 0} k p_{k}<\infty$. Starting from their point of creation on the path of their parent, each of these children moves and reproduces according to an independent copy of the triple $(B, \eta, X)$. Note that there is always at least one offspring guaranteed and hence the process survives with probability one.

In this text we shall use the Ulam-Harris labelling notation such that an individual $u$ is identified by its line of decent from the initial ancestor. That is, if $u=\left(\emptyset, i_{1}, \ldots, i_{n-1}, i_{n}\right)$ then she is the $i_{n}$ th child of the $i_{n-1}$ th child of $\ldots$ of the $i_{1}$ th child of the initial ancestor, $\varnothing$. Thus $u v$ refers to the individual who, from $u$ 's perspective, has line of descent expressed as $v$. Let $Z_{t}$ be the point process describing the number and positions of individuals alive at time $t,\left\{\Xi_{u}(t): u \in N_{t}\right\}$ where $N_{t}$ is the set of individuals alive at time $t$.

Let $\mathcal{F}_{t}=\sigma\left(Z_{s}: s \leqslant t\right), P$ be the law of the branching Brownian motion as it is defined above and $E$ be expectation with respect to $P$. A natural $P$-martingale with respect to the filtration $\mathcal{F}_{t}$ that arises in branching Brownian motion takes the form

$$
W_{t}(\lambda):=\sum_{u \in N_{t}} e^{-\lambda\left(\Xi_{u}(t)+c_{\lambda} t\right)}
$$

for $t$ and $\lambda$ positive with $c_{\lambda}=\lambda / 2+\beta m / \lambda$. See Chauvin [14], Kingman [30], Biggins [6] and Neveu [39] for further details. From these references one can find the following theorem, or similar versions of it.

Theorem 1. Let $\underline{\lambda}:=\sqrt{2 \beta m}$ so that $c_{\lambda}$ attains a local minimum and local maximum at $\pm \underline{\lambda}$. The limit $W(\lambda):=$ $\lim _{t \uparrow \infty} W_{t}(\lambda)$ exists $P$-almost surely and

(i) if $|\lambda|>\underline{\lambda}$ then $W(\lambda)=0$ P-almost surely,

(ii) if $|\lambda|=\underline{\lambda}$ then $W(\lambda)=0 P$-almost surely,

(iii) if $|\lambda| \in[0, \underline{\lambda})$ then $W(\lambda)=0$-almost surely or $W(\lambda)$ is an $L^{1}(P)$-limit accordingly as $E\left(X \log ^{+} X\right)=\infty$ or $E\left(X \log ^{+} X\right)<\infty$.

Remark 2. In contrast to part (iii) above note that for the case of dyadic branching (binary splitting) with unit branching rate, Neveu [39] establishes $L^{p}(P)$ convergence for $p \in(1,2]$ provided that $p \lambda^{2} / 2 \leqslant 1$.

It is also known that the negative derivative of $W_{t}(\lambda)$,

$$
\partial W_{t}(\lambda):=-\frac{\partial}{\partial \lambda} W_{t}(\lambda)=\sum_{u \in N_{t}}\left(\Xi_{u}(t)+\lambda t\right) e^{-\lambda\left(\Xi_{u}(t)+c_{\lambda} t\right)},
$$

is a signed $P$-martingale with respect to $\mathcal{F}_{t}$ (it is also easy to check) which we shall refer to as the derivative martingale. As far as the author is aware, the following theorem concerning this martingale strengthens existing results for branching Brownian motion. The result shall appear as a by-product of subsequent analysis in this article.

Theorem 3. For all $|\lambda| \geqslant \underline{\lambda}, \partial W(\lambda):=\lim _{t \uparrow \infty} \partial W_{t}(\lambda)$ exists $P$-almost surely. Further,

(i) if $|\lambda|>\underline{\lambda}$ then $\partial W(\lambda)=0$ almost surely,

(ii) if $|\lambda|=\underline{\lambda}$ then $\partial W(\lambda)=0$ when $E\left(X\left(\log ^{+} X\right)^{2-\delta}\right)=\infty$ for some $\delta>0$ or $\partial W(\lambda) \in(0, \infty)$ (respectively $\partial W(\lambda) \in(-\infty, 0)) P$-almost surely when $\lambda>0$ (respectively $\lambda<0)$ and $E\left(X\left(\log ^{+} X\right)^{2+\delta}\right)<\infty$ for some $\delta>0$. 
For the case of dyadic (binary splitting) branching Brownian motion, the result in part (ii) of this theorem was proved for $|\lambda|=\underline{\lambda}$ by both Neveu [39] and Harris [26]; both using an application of solutions to a certain travelling wave equation which we shall shortly describe.

Remark 4. The theorem above is lacking a statement for the regime $|\lambda| \in[0, \underline{\lambda})$. Unfortunately it seems difficult to see how the methods used in this text may apply to this case. The point of failure of the current methods is pointed out in the discussion following Theorem 9 in Section 4. Results in this regime are however available in $[7,8]$ where it is proved that under appropriate moment conditions $\partial W(\lambda)$ exists as a limit almost surely and, for example, in $L^{1}(P)$ (as a consequence of $W(\cdot)$ being analytic on a suitable domain).

Remark 5. The moment conditions in Theorem 3 have a 'gap' in the sense that it is not clear what happens when for example $E\left(X\left(\log ^{+} X\right)^{2}\right)<\infty$. A similar result to that of Theorem 3 was developed in [10] for the branching random walk. In that case however, the moment conditions are much sharper than those given here although still have a 'gap'. In principle one could use the methods there to sharpen the moments given here.

Interest in the limit of these martingales is stimulated by its intimate connection with travelling wave solutions to the Kolmogorov-Petrovskii-Piskounov equation

$$
\frac{\partial u}{\partial t}=\frac{1}{2} \frac{\partial^{2} u}{\partial x^{2}}+\beta(f(u)-u)
$$

where $f(u)=E\left(s^{X+1}\right)$, taking solutions $u: \mathbb{R} \times \mathbb{R}^{+} \rightarrow[0,1]$. This reaction-diffusion equation has been studied by many authors, both probabilistically and analytically (see, for example, Kolmogorov et al. [31], Fisher [23], Skorohod [43], McKean [38], Bramson [11,12], Neveu [39], Uchiyama [44], Aronson and Weinburger [1], Karpelevich et al. [28] and Kelbert and Suhov [29] to name but a few).

Of particular interest however is the recent exposition of Harris [26] who, using probabilistic arguments alone, gives an elegant derivation of the existence, uniqueness and asymptotics of travelling wave solutions to (1). By a travelling wave solution it is meant a twice continuously differentiable, monotone increasing function $\Phi_{c}: \mathbb{R} \rightarrow[0,1]$ such that $\Phi_{c}(-\infty)=0=1-\Phi_{c}(\infty)$ with $u(x, t)=\Phi_{c}(x-c t)$ a solution to $(1) ; c \in \mathbb{R}$ is the wave speed. Substituting into (1) shows that $\Phi_{c}$ solves the ordinary differential equation

$$
\frac{1}{2} \Phi_{c}^{\prime \prime}+c \Phi_{c}^{\prime}+\beta\left(f\left(\Phi_{c}\right)-\Phi_{c}\right)=0
$$

In the sequel, when talking of (non-trivial) travelling waves, we shall always mean in the sense described above. Otherwise, it is possible to talk of travelling waves which, for example, are not monotone and bounded in [0, 1]; cf. McKean [38] or Kolmogorov et al. [31]. We wish to exclude these travelling waves from this discussion. It is also worth pointing out that Eq. (2) always admits the trivial solutions which are the functions that are identically 0 and identically 1 . Note also that if $\Phi_{c}(x)$ is a travelling wave with wave speed $c$ then so is $\Phi_{c}(x+y)$ for any $y \in R$. Uniqueness can thus in principle only be established up to a spatial shift.

We shall offer in this paper a proof of the existence, asymptotics and uniqueness of the above mentioned travelling wave solutions to (1) using again purely probabilistic methods but none the less of a different flavour to those of Harris [26]. Indeed we shall demonstrate the following results.

\section{Kolmogorov-Petrovskii-Piskounov travelling waves.}

Subcriticality. Travelling waves do not exist when $|c|<\underline{c}:=\sqrt{2 \beta m}=\underline{\lambda}$.

Criticality. When $|c|=\underline{c}$ and $E\left(X\left(\log ^{+} X\right)^{2+\delta}\right)<\infty$ for some $\delta>0$ then (modulo an additive constant in the argument) there is a unique travelling wave at speed $\underline{c}$ given by

$$
\Phi_{\underline{c}}(x)=E\left(\exp \left\{-e^{-\underline{\lambda} x} \partial W(\underline{\lambda})\right\}\right) .
$$


Further this unique travelling wave has the asymptotic

$$
1-\Phi_{\underline{c}}(x) \sim \text { const } \times x e^{-\underline{\lambda} x}
$$

as $x$ tends to infinity.

Supercriticality. When $|c|>\underline{c}$ and $E\left(X \log ^{+} X\right)<\infty$ then (modulo an additive constant in the argument) there is a unique travelling wave at speed $c$ given by

$$
\Phi_{c_{\lambda}}(x)=E\left(\exp \left\{-e^{-\lambda x} W(\lambda)\right\}\right)
$$

where $|\lambda| \in[0, \underline{\lambda})$ such that $c=c_{\lambda}$. Further this unique travelling wave has the asymptotic

$$
1-\Phi_{c_{\lambda}}(x) \sim \text { const } \times e^{-\lambda x}
$$

as $x$ tends to infinity.

Remark 6. The summary above is of course not complete as one may still ask what happens at criticality when $E\left(X\left(\log ^{+} X\right)^{2+\delta}\right)=\infty$ for all $\delta>0$ and at supercriticality when $E\left(X \log ^{+} X\right)=\infty$. In the latter case, Biggins and Kyprianou [9] have shown that (for example) when $m=E(X)<\infty$ there exist Seneta-Heyde norming constants, say $\left\{c_{t}: t \geqslant 0\right\}$, so that there exists a limit in probability of the sequence $\left\{c_{t} W_{t}(\lambda): t \geqslant 0\right\}$ whose Laplace transform is a non-trivial travelling wave. It is then possible to construct arguments based on those of Biggins and Kyprianou [9] to show that this non-trivial travelling wave is in fact unique and further has the same asymptotic as above. The issue of existence at criticality in the case that $E\left(X\left(\log ^{+} X\right)^{2+\delta}\right)=\infty$ is somewhat less clear. It might be conjectured that one could produce a Seneta-Heyde norming of the martingale $W$ in this case whose limit will provide existence of a travelling. Indeed embedded in the results of [5] is the existence of a Seneta-Heyde norming sequence for an analogue of the martingale $W$ under an $x \log x$ moment condition and within the context of a special class of branching random walk. In this case the limit again has Laplace transform which provides a solution to an analogue of the travelling wave equation.

The main difference in the probabilistic approach that we use here when comparing with [26] is that we first establish non-trivial martingale limits $W$ and $\partial W$ from which existence, uniqueness and asymptotics follow. Harris relied on classical 'single particle' martingale representations of the travelling wave equation using the FeynmanKac formula to first achieve asymptotics. Once the asymptotics had been established Harris then applies them together with simple probabilistic considerations to recover convergence of $\partial W$ and uniqueness of the travelling waves at critical and supercritical wave speeds. Note also that Harris [26] also only considers dyadic branching.

Here we appeal to 'spine decompositions' in order to prove martingale convergence, similar versions of which have appeared in $[36,35,22]$ for superprocesses. Note that in the latter reference the decomposition is known as the 'immortal particle picture' and concerns processes which become extinct with probability one. The spine decomposition given here is essentially the one given in [15, p. 306]. Other examples of spine decompositions for a variety of branching particle processes can be found in $[17,3,32,40]$. For the critical case, we work with a decomposition in which the spine has a conditioned behaviour. Part of the work here was inspired by and has inspired methods in [10] for the branching random walk where the techniques work equally well. The methods used here to deduce martingale convergence have also been used successfully in [20] for related martingales that appear naturally for more general branching Markov diffusions. Consideration of martingale convergence and conditioned spine decomposition there lead to probabilistic proofs of results concerning local extinction/survival properties.

The idea of a branching particle process being intimately linked to a certain class of travelling waves is not a story that is unique to branching Brownian motion. Of the few other examples that can be seen in the literature, one should consult $[13,34]$ where a coupled system of reaction-diffusion equations are linked to a two type branching diffusion and $[24,25]$ where it can be seen that travelling waves to a diffusion equation with two spatial parameters and a spatially dependent branching rate exists. For branching random walks, one has a functional equation in place 
of the travelling wave equation, see [6]. In contrast, the analogous relationship between superprocesses and certain non-linear differential equations has received more attention; see, for example, the overviews in $[21,33,19]$.

In what follows and for the remainder of the paper we shall deal only with the case that $c \geqslant 0$ and $\lambda \geqslant 0$ unless otherwise stated. Travelling waves with negative wave speeds can be analyzed by simple considerations of symmetry.

\section{Proof of Theorem 1 and existence of supercritical travelling waves}

\subsection{Spines}

For future use we shall recall some standard Radon-Nikodym derivatives for measures we shall be interested in. Let $\mathbb{L}^{(\alpha)}$ be the law of a Poisson process $n=\left(\left\{v_{i}: i=1, \ldots, n_{t}\right\}: t \geqslant 0\right)$ with rate $\alpha>0$ that is adapted to some filtration $\left\{\mathcal{G}_{t}: t \geqslant 0\right\}$. Let $\mathbb{L}_{t}^{(\alpha)}$ be its restriction to $\mathcal{G}_{t}$. We have

$$
\frac{d \mathbb{L}_{t}^{(\beta(m+1))}}{d \mathbb{L}_{t}^{(\beta)}}(n)=e^{-\beta m t}(m+1)^{n_{t}}
$$

for all $t>0$. Define $\mathbb{P}^{-\lambda}$ to be the law under which $B$ is a Brownian motion with drift $-\lambda$, where $\lambda \in \mathbb{R}$. Assume that $B$ is adapted to some filtration $\left\{\mathcal{H}_{t}: t \geqslant 0\right\}$. Let $\mathbb{P}_{t}^{-\lambda}$ be its restriction to $\mathcal{H}_{t}$ so that

$$
\frac{d \mathbb{P}_{t}^{-\lambda}}{d \mathbb{P}_{t}}(B)=e^{-\lambda B(t)-\lambda^{2} t / 2}
$$

for all $t>0$ where $\mathbb{P}=\mathbb{P}^{0}$. Finally let $\left(\tilde{p}_{k}: k \geqslant 0\right)$ be the tilted distribution for $X$ such that $\tilde{p}_{k}=(k+1) p_{k} /(m+1)$ for all $k \geqslant 0$.

Now let $\left(\mathcal{T}, \mathcal{F}, \mathcal{F}_{t}, P\right)$ be the filtered probability space in which the branching Brownian motion $\left\{Z_{t}: t \geqslant 0\right\}$ is defined. The symbol $\mathcal{T}$ denotes the space of Galton-Watson trees with nodes having marks in $\mathbb{R}^{+} \times C\left(\mathbb{R}^{+}, \mathbb{R}\right)$ (where $C\left(\mathbb{R}^{+}, \mathbb{R}\right)$ is the space of continuous maps from $\mathbb{R}^{+}$to $\mathbb{R}$ ) which are realizations of their life length and spatial path whilst alive relative to their birth position and $\mathcal{F}$ may be taken as $\sigma(\mathcal{T})$.

To be more specific, let $\mathbb{T}$ be the space of Galton-Watson trees. A Galton-Watson tree $\tau \in \mathbb{T}$ is a point in the space of possible Ulam-Harris labels

$$
\Omega=\emptyset \cup \bigcup_{n \in \mathbb{N}^{+}}(\mathbb{N})^{n}
$$

where $\mathbb{N}=\{1,2,3, \ldots\}$ such that

(i) $\varnothing \in \tau$ (the initial ancestor),

(ii) if $u, v \in \Omega$, the concatenation $u v \in \tau$ implies $u \in \tau$,

(iii) for all $u \in \tau$, there exists $X_{u} \in\{0,1,2, \ldots\}$ so that when $j \in \mathbb{N}, u j \in \tau$ if and only if $1 \leqslant j \leqslant 1+X_{u}$.

Each individual $u \in \tau$ has mark $\left(\eta_{u}, B_{u}\right) \in \mathbb{R}^{+} \times C\left(\mathbb{R}^{+}, \mathbb{R}\right)$ where $B_{u}=\left\{B_{u}(s): s \in\left[0, \eta_{u}\right)\right\}$ is the motion of $u$ relative to its birth position whilst alive and $\eta_{u}$ is the life length of $u$ at which point it undergoes fission. With this notation we have that the moment of death of individual $u$ can be written as $v_{u}=\sum_{v \leqslant u} \eta_{v}$, the moment of birth $b_{u}=\sum_{v<u} \eta_{v}$ (an empty sum is zero) and the position of $u$ when alive at time $t$ is given by $\Xi_{u}(t)=\sum_{v<u} B_{v}\left(\eta_{v}\right)+B_{u}\left(t-b_{u}\right)$. In this way we may identify, for example, our earlier notation as

$$
N_{t}=\left\{u \in \tau: b_{u} \leqslant t<v_{u}\right\} \quad \text { and } \quad Z_{t}(\cdot)=\sum_{u \in N_{t}} \delta_{\Xi_{u}(t)}(\cdot)
$$


with $\delta_{x}$ as the usual unit point measure at $x \in \mathbb{R}$.

We shall write $(\tau, B, \eta)$ as short hand for the marked Galton-Watson tree $\left\{\left(u, \eta_{u}, B_{u}\right): u \in \tau\right\}$ so that $\mathcal{T}=\{(\tau, B, \eta): \tau \in \mathbb{T}\}$. The sigma-algebra $\mathcal{F}_{t}$ is generated by

$$
\left\{\begin{array}{l}
\left(u, X_{u}, \eta_{u},\left\{B_{u}(s): s \in\left[0, \eta_{u}\right]\right\}: u \in \tau \text { with } v_{u} \leqslant t\right) \text { and } \\
\left(u,\left\{B_{u}(s): s \in\left[0, t-b_{u}\right]\right\}: u \in \tau \text { with } t \in\left[b_{u}, v_{u}\right)\right): \tau \in \mathbb{T} .
\end{array}\right\}
$$

The measure $P$ corresponds to the unique law such that individuals live for independent exponential lengths of time at the end of which they produce a number of offspring independently distributed like $1+X$ which are positioned at the spatial point of death of their parent and whose motion whilst alive is that of an independent Brownian motion. (A similar description of a branching Brownian motion to this has appeared for example in [15,16].) We shall use $P_{t}$ for the restriction of $P$ to $\mathcal{F}_{t}$.

For any $\tau \in \mathbb{T}$ we can identify distinguished genealogical lines of descent from the initial ancestor each of which shall be referred to as a spine. An identified spine will be written $\xi=\left\{\xi_{0}=\emptyset, \xi_{1}, \xi_{2}, \ldots\right\}$ where $\xi_{n} \in \tau$ is the label of $\xi$ 's node in the $n$th generation. We shall write $u \in \xi$ to mean that $u=\xi_{i}$ for some $i \geqslant 0$. Now let

$$
\widetilde{\mathcal{T}}=\{(\tau, B, \eta, \xi): \xi \subseteq \tau \in \mathbb{T}\}
$$

be the enriched space of marked trees in $\mathcal{T}$ with distinguished spine, $\xi$, let $\widetilde{\mathcal{F}}=\sigma(\widetilde{\mathcal{T}})$ and

$$
\widetilde{\mathcal{F}}_{t}=\sigma\left(\mathcal{F}_{t},\left\{(\xi: u \in \xi): u \in N_{t}\right\}\right)
$$

the sigma-algebra generated by $\mathcal{F}_{t}$ and sets of spines which are characterized by their common genealogy up to time $t$. We shall denote $\Xi=\{\Xi(t): t \geqslant 0\}$ the spatial path followed by a spine and write $n=\left\{n_{t}: t \geqslant 0\right\}$ for the counting process of points of fission along the spine. Both $\Xi$ and $n$ can be constructed from $(\tau, B, \eta, \xi)$. Indeed, if a spine contains the node $u \in N_{t}$ then in accordance with previous notation, $\Xi(t)=\Xi_{u}(t)$, which is ultimately recovered from the marks $B$. Further, $n_{t}=|u|$ and $\left\{v_{v}: v<u\right\}$ are the times of fission along the spine until time $t$, all of which can be recovered from the marks $\eta$. Also at the fission time corresponding to node $v$ there are $X_{v}$ independent new marked trees rooted at the space time point $\left(\Xi\left(v_{v}\right), v_{v}\right)$ growing off the spine, say $\left\{(\tau, B, \eta)_{j}^{v} \in \mathcal{T}: 1 \leqslant j \leqslant X_{v}\right\}$.

We construct the non-probability measure $P_{t}^{*}$ on $\left(\widetilde{\mathcal{T}}, \widetilde{\mathcal{F}}_{t}\right)$ such that

$$
d P_{t}^{*}(\tau, B, \eta, \xi)=d \mathbb{P}_{t}(\Xi) d \mathbb{L}_{t}^{(\beta)}(n) \prod_{v<\xi_{n_{t}}} p_{X_{v}} \prod_{j=1}^{X_{v}} d P_{t-v_{v}}\left((\tau, B, \eta)_{j}^{v}\right)
$$

(empty products are taken as one). Note that $p_{X_{v}}=\sum_{k \geqslant 0} p_{k} \mathbf{1}_{\left(X_{v}=k\right)}$ is the probability that individual $v$ has $1+X_{v}$ offspring. For a given $\widetilde{\mathcal{F}}_{t}$-measurable test function of the form

$$
f(\tau, B, \eta, \xi)=\sum_{u \in N_{t}} f_{u}(\tau, B, \eta) \mathbf{1}_{(u \in \xi)}
$$

where $f_{u}(\tau, B, \eta)$ is $\mathcal{F}_{t}$-measurable, we have the following decomposition

$$
\begin{aligned}
\int_{\widetilde{\mathcal{T}}} f(\tau, B, \eta, \xi) d P_{t}^{*}(\tau, B, \eta, \xi) & =\int_{\widetilde{\mathcal{T}}} \sum_{u \in N_{t}} f_{u}(\tau, B, \eta) \mathbf{1}_{(u \in \xi)} d P_{t}^{*}(\tau, B, \eta, \xi) \\
& =\int_{\mathcal{T}} \sum_{u \in N_{t}} f_{u}(\tau, B, \eta) d P_{t}(\tau, B, \eta) .
\end{aligned}
$$

Note in particular then that the total mass of $P_{t}^{*}$ is given by

$$
P_{t}^{*}(\widetilde{\mathcal{T}})=\int_{\widetilde{\mathcal{T}}} \sum_{u \in N_{t}} \mathbf{1}_{(u \in \xi)} d P_{t}^{*}(\tau, B, \eta, \xi)=\int_{\mathcal{T}}\left|N_{t}\right| d P_{t}(\tau, B, \eta)=e^{m \beta t}
$$


where $\left|N_{t}\right|=Z_{t}(\mathbb{R})$ is the cardinality of the set of individuals alive at time $t$. The last equality is well known for Markov branching processes, see, for example, [2].

Consider also the bivariate measure $\pi_{t}^{*}$ on $\left(\widetilde{\mathcal{T}}, \widetilde{\mathcal{F}}_{t}\right)$ given by

$$
\begin{aligned}
d \pi_{t}^{*}(\tau, B, \eta, \xi) & =e^{-\lambda\left(\Xi(t)+c_{\lambda} t\right)} d P_{t}^{*}(\tau, B, \eta, \xi) \\
& =e^{-\lambda \Xi(t)-\lambda^{2} t / 2} e^{-\beta m t}(m+1)^{n_{t}} \prod_{v<\xi_{n_{t}}}\left(\frac{X_{v}+1}{m+1}\right) \frac{1}{X_{v}+1} d P_{t}^{*}(\tau, B, \eta, \xi) \\
& =d \mathbb{P}_{t}^{-\lambda}(\Xi) d \mathbb{L}_{t}^{(\beta(m+1))}(n) \prod_{v<\xi_{n_{t}}}\left(\frac{X_{v}+1}{m+1}\right) p_{X_{v}} \frac{1}{X_{v}+1} \prod_{j=1}^{X_{v}} d P_{t-v_{v}}\left((\tau, B, \eta)_{j}^{v}\right)
\end{aligned}
$$

inducing a measure $\pi^{*}$ on $(\tilde{\mathcal{T}}, \tilde{\mathcal{F}})$. Using (7) we can perform a similar calculation to (8) to check that $\pi_{t}^{*}$ and hence $\pi^{*}$ are probability measures;

$$
\begin{aligned}
\int_{\widetilde{\mathcal{T}}} d \pi_{t}^{*}(\tau, B, \eta, \xi) & =\int_{\widetilde{\mathcal{T}}} e^{-\lambda\left(\Xi(t)+c_{\lambda} t\right)} d P_{t}^{*}(\tau, B, \eta, \xi) \\
& =\int_{\widetilde{\mathcal{T}}} \sum_{u \in N_{t}} e^{-\lambda\left(\Xi_{u}(t)+c_{\lambda} t\right)} \mathbf{1}_{(u \in \xi)} d P_{t}^{*}(\tau, B, \eta, \xi) \\
& =\int_{\mathcal{T}} W_{t}(\lambda) d P_{t}(\tau, B, \eta)
\end{aligned}
$$

which equals one on account of $W_{t}(\lambda)$ being a normalized martingale. Further, we can marginalize $\pi_{t}^{*}$ to $\left(\mathcal{T}, \mathcal{F}_{t}\right)$ giving a probability measure $\pi_{t}(t \geqslant 0)$ which we claim satisfies

$$
\frac{d \pi_{t}}{d P_{t}}=W_{t}(\lambda)
$$

thus inducing a measure $\pi$ on $(\mathcal{T}, \mathcal{F})$. To see why this claim is true note that for any $\mathcal{F}_{t}$-measurable test function $g(\tau, B, \eta)$ we can argue again via the formula (7) in the following way;

$$
\begin{aligned}
\int_{\mathcal{T}} g(\tau, B, \eta) d \pi_{t}(\tau, B, \eta) & =\int_{\widetilde{\mathcal{T}}} g(\tau, B, \eta) d \pi_{t}^{*}(\tau, B, \eta, \xi) \\
& =\int_{\widetilde{\mathcal{T}}} g(\tau, B, \eta) \sum_{u \in N_{t}} \mathbf{1}_{(u \in \xi)} d \pi_{t}^{*}(\tau, B, \eta, \xi) \\
& =\int_{\widetilde{\mathcal{T}}} g(\tau, B, \eta) \sum_{u \in N_{t}} e^{-\lambda\left(\Xi_{u}(t)+c_{\lambda} t\right)} \mathbf{1}_{(u \in \xi)} d P_{t}^{*}(\tau, B, \eta, \xi) \\
& =\int_{\mathcal{T}} g(\tau, B, \eta) W_{t}(\lambda) d P_{t}(\tau, B, \eta) .
\end{aligned}
$$

In view of the Radon-Nikodym derivatives outlined at the beginning of this section, the construction in (9) shows that the process $\left\{Z_{t}: t \geqslant 0\right\}$ under $\pi$ corresponds to the law of a non-homogeneous branching motion with distinguished and randomized spine having the following properties:

(i) the diffusion along the spine begins from the origin of space and time and moves according to a Brownian motion with drift $-\lambda$, 
(ii) points of fission along the spine are independent of its motion and occur with accelerated rate $(m+1) \beta$,

(iii) the distribution of offspring numbers at each point of fission on the spine is independent of spinal motion and times of reproduction and has tilted measure $\left(\tilde{p}_{k}: k \geqslant 0\right)$,

(iv) the spine is chosen randomly so that at each fission point the next individual to represent the spine is chosen with uniform probability from the offspring of the current representative,

(v) offspring of individuals on the spine which are not part of the spine initiate $P$-branching Brownian motions at their space-time point of creation.

This decomposition was first established in [15].

\subsection{Proof of Theorem 1}

Using the change of measure (10) we can recover the known necessary and sufficient conditions on $\lambda$ and $X$ that imply $L^{1}(P)$-convergence of $W_{t}(\lambda)$. Essential to the argument is the following fundamental measure theoretic result (see, for example, [18, p. 242], [3]). Let $\bar{W}(\lambda)=\limsup _{t \uparrow \infty} W_{t}(\lambda)$ so that $\bar{W}(\lambda)=W(\lambda) P$-a.s., then

$$
\begin{array}{ll}
\bar{W}(\lambda)=\infty \pi \text {-a.s. } & \Longleftrightarrow \bar{W}(\lambda)=0 P \text {-a.s. } \\
\bar{W}(\lambda)<\infty \pi \text {-a.s. } \quad \Longleftrightarrow \quad \int \bar{W}(\lambda) d P=1 .
\end{array}
$$

We proceed now with the proofs of the three parts of Theorem 1 in a similar manner to [35] and [36].

(i)-(ii) Suppose that $\lambda \geqslant \underline{\lambda}$. We have $c_{\lambda} \leqslant \lambda$ so that $\left\{\Xi(t)+c_{\lambda} t: t \geqslant 0\right\}$ is a $\pi^{*}$-Brownian motion with nonpositive drift. As

$$
W_{t}(\lambda) \geqslant \exp \left\{-\lambda\left(\Xi(t)+c_{\lambda} t\right)\right\}
$$

it follows immediately that $\bar{W}(\lambda)=\infty \pi^{*}$-a.s. and hence by $(11), W(\lambda)=0 P$-a.s.

(iii) We begin the proof of this part by making some remarks on the finiteness of $E\left(X \log ^{+} X\right)$. A sequence of simple calculations shows that $E\left(X \log ^{+} X\right)$ is (in)finite if and only if

$$
\sum_{k \geqslant 1} \tilde{\operatorname{Pr}}(\log X>c k)
$$

is (in)finite for any $c>0$ where under $\widetilde{\operatorname{Pr}}, X$ has the tilted distribution $\left\{\tilde{p}_{k}: k \geqslant 0\right\}$. Thus if under $\widetilde{\operatorname{Pr}},\left\{X_{n}: n \geqslant 0\right\}$ is a sequence of independent copies of $X$, then (by the Borel-Cantelli lemma)

$$
\lim \sup _{t \uparrow \infty} n^{-1} \log X_{n}= \begin{cases}0 & \text { if } E\left(X \log ^{+} X\right)<\infty \\ \infty & \text { if } E\left(X \log ^{+} X\right)=\infty .\end{cases}
$$

Pr-almost surely.

Now suppose that $\lambda \in[0, \underline{\lambda})$ and $E\left(X \log ^{+} X\right)=\infty$. The motion along the spine $\left\{\Xi(t)+c_{\lambda} t: t \geqslant 0\right\}$ is a $\pi^{*}$-Brownian motion with strictly positive drift. Let $\left\{\nu_{\xi_{i}}: i \geqslant 0\right\}$ be the times of fission along the spine $\xi$. Since

$$
W_{v_{\xi_{k}}}(\lambda) \geqslant X_{\xi_{k}} \exp \left\{-\lambda\left(\Xi\left(\nu_{\xi_{k}}\right)+c_{\lambda} \nu_{\xi_{k}}\right)\right\}
$$

the Strong Law of Large Numbers together with the behaviour of the sequences $\left\{X_{\xi_{k}}: k \geqslant 0\right\}$ and $\left\{\Xi(t)+c_{\lambda} t: t \geqslant\right.$ $0\}$ yields that $\bar{W}(\lambda)=\infty \pi^{*}$-a.s. and hence once again by $(11), W(\lambda)=0 P$-a.s.

Finally suppose that $\lambda \in[0, \underline{\lambda})$ and $E\left(X \log ^{+} X\right)<\infty$. Define $\mathcal{G}$ to be the sigma-algebra generated by the genealogy along the spine, the diffusion on the spine $\Xi$, the Poisson process representing the birth times along the spine $n$ and $\left\{X_{\xi_{i}}: i \geqslant 0\right\}$. A brief computation, based on the decomposition of $W_{t}(\lambda)$ according to contributions from descendents of individuals born along the spine, yields

$$
E_{\pi^{*}}\left(W_{t}(\lambda) \mid \mathcal{G}\right)=\sum_{i=1}^{n_{t}} X_{\xi_{i-1}} e^{-\lambda\left(\Xi\left(v_{\xi_{i-1}}\right)+c_{\lambda} \nu_{\xi_{i-1}}\right)}+e^{-\lambda\left(\Xi(t)+c_{\lambda} t\right)} .
$$


Recall that within the specified regime of $\lambda,\left\{\Xi(t)+c_{\lambda} t: t \geqslant 0\right\}$ is a $\pi^{*}$-Brownian motion with strictly positive drift. (Note however that when $\lambda=0$, the summands in (13) are simply $X_{\xi_{i-1}} e^{-\beta m t}$.) The moment condition ensures that extremes of the sequence of variables $\left\{X_{\xi_{i}}: i \geqslant 0\right\}$ have sub-exponential behaviour. Consequently (again using the Law of Large Numbers)

$$
\underset{t \uparrow \infty}{\limsup } E_{\pi^{*}}\left(W_{t}(\lambda) \mid \mathcal{G}\right)<\infty \quad \pi^{*} \text {-a.s. }
$$

Fatou's lemma now tells us that $\liminf _{t \uparrow \infty} W_{t}(\lambda)<\infty \pi$-a.s. In light of $(10), W_{t}(\lambda)^{-1}$ is a $\pi$-martingale with an almost sure limit and thus by the previous sentence, $\lim _{t \uparrow \infty} W_{t}(\lambda)<\infty \pi$-a.s. We thus conclude from (12) that for $\lambda \in[0, \underline{\lambda})$ and $E\left(X \log ^{+} X\right)<\infty, W_{t}(\lambda)$ converges $P$-almost surely and in $L^{1}(P)$.

\subsection{Existence at supercriticality}

Existence at supercritical wave speeds in the regime $c_{\lambda}>\underline{c}, \lambda \in[0, \underline{\lambda})$ and $E\left(X \log ^{+} X\right)<\infty$ follows readily. To see this it suffices to follow the reasoning of Harris [26] or Biggins and Kyprianou [9] as below.

We can easily make the decomposition for all $t>s>0$,

$$
W_{t}(\lambda) \stackrel{d}{=} \sum_{u \in N_{s}} e^{-\lambda\left(\Xi_{u}(s)+c_{\lambda} s\right)} W_{t-s}(\lambda, u)
$$

where $W_{t-s}(\lambda, u)$ are independent copies of $W_{t-s}(\lambda)$ for each $u \in N_{s}$. Letting $t$ tend to infinity and taking an exponentially rescaled Laplace transform of the resulting identity yields the functional equation

$$
\Phi(x)=E\left[\prod_{u \in N_{s}} \Phi\left(x+\Xi_{u}(s)+c_{\lambda} s\right)\right]
$$

for all $s>0$, where $\Phi(x)=E\left[\exp \left\{-e^{-\lambda x} W(\lambda)\right\}\right]$. It is known that $\Phi$ solves the functional equation (15) if and only if it solves the ODE (2) with wave speed $c_{\lambda}$; see, for example, [38,13]. Existence of a non-trivial travelling wave as we have defined it in the introduction would be established were it not for the fact that it is not clear whether $\Phi(-\infty)=0$ on account of the fact that we have not proved that $p:=P(W(\lambda)=0)=0$. (Note monotonicity, boundedness and the fact that $\Phi(\infty)=0$ can all be trivially checked.) However by taking limits in (14), it is clear from the distributional identity that follows that $p$ must satisfy $p=E\left(p^{Z_{s}(\mathbb{R})}\right)$ for all $s>0$. Thanks to the Markov and branching property, it is easily confirmed that $\left\{p^{Z_{t}(\mathbb{R})}: t \geqslant 0\right\}$ is a martingale. Since $p<1$, the limit of this latter martingale will be zero, contradicting the Martingale Convergence theorem unless $p=0$. The proof of existence is now complete.

Remark 7. In this paper we do not offer new probabilistic non-existence of travelling waves for wave speeds less than $\underline{c}$. For completeness we quickly recall the martingale argument given in [26]. Let $L_{t}=\inf \left\{\Xi_{u}(t): u \in N_{t}\right\}$. A classic result for branching Brownian motion says that $\lim _{t \uparrow \infty} L_{t} / t=\underline{c}$. It follows that $\lim _{t \uparrow \infty} L_{t}+c t=$ $-\infty$ for all $c<\underline{c}$. For any $c<\underline{c}$, a non-trivial travelling wave $\Phi_{c}$ produces a $P$-martingale of the form $\prod_{u \in N_{t}} \Phi_{c}\left(\Xi_{u}(t)+c t\right)$ which has an almost sure and $L^{1}(P)$ limit (see, for example, [14]). However this martingale is bounded above by $\Phi_{c}\left(L_{t}+c t\right)$ which tends to zero on account of the previous remark and that, per definition, $\Phi_{c}(-\infty)=0$. Thus we reach a contradiction leading to the conclusion that no (bounded) travelling wave exists.

\section{Martingales on stopping lines, asymptotics and uniqueness at supercriticality}

We remain in the regime $c_{\lambda}>\underline{c}, \lambda \in[0, \underline{\lambda})$ and $E\left(X \log ^{+} X\right)<\infty$. On the space-time half plane $\{(y, t): y \in$ $\left.\mathbb{R}, t \in \mathbb{R}^{+}\right\}$, consider the barrier $\Gamma^{\left(x, c_{\lambda}\right)}$ described by the line $y+c_{\lambda} t=x$ for $x>0$. By arresting lines of descent the first time they hit this barrier we produce a random collection of individuals, $C\left(x, c_{\lambda}\right)$, which is a stopping line. 
That is to say they have the fundamental property of a general stopping line $\mathcal{L}$ that $u \in \mathcal{L}$ if and only if $v \notin \mathcal{L}$ for all $v<u$. What is important to note for our purposes are the following known facts.

(i) On account of the fact that $W(\underline{\lambda})=0$ it follows by looking at the largest summand of $W_{t}(\underline{\lambda})$ that $\lim _{t \uparrow \infty} L_{t}+\underline{c} t=\infty$ and hence $\lim _{t \uparrow \infty} L_{t}+c_{\lambda} t=\infty$ in the regime $\lambda \in[0, \underline{\lambda}]$. An elementary argument by contradiction now shows that all lines of descent from the initial ancestor will hit $\Gamma^{\left(x, c_{\lambda}\right)}$ with probability one for all $x>0$; in this sense we call the sequence $\left\{C\left(x, c_{\lambda}\right): x \geqslant 0\right\}$ dissecting.

(ii) We have $\lim _{x \uparrow \infty} \inf \left\{|u|: u \in C\left(x, c_{\lambda}\right)\right\}=\infty$. This follows from the fact that the number of individuals in the $n$th generation are almost surely finite, their life lengths are almost surely finite and hence the space-time point of the right most extreme reached by any individual in the $n$th generation must also be almost surely finite. This means that for each $n$, one can choose $x$ sufficiently large so that $\inf \left\{|u|: u \in C\left(x, c_{\lambda}\right)\right\}>n$. In this sense we say that the sequence $\left\{C\left(x, c_{\lambda}\right): x \geqslant 0\right\}$ is tending to infinity as $x$ tends to infinity.

(iii) The cardinality process $\left\{\left|C\left(x, c_{\lambda}\right)\right|: x \geqslant 0\right\}$ forms a (supercritical) continuous time branching process ( $x$ plays the role of time). This result is due to Neveu [39]. The observation follows from the Strong Markov Branching Property, found, for example, in [14]. For our purposes, the Strong Markov Branching Property says that if $\left\{\sigma_{u}: u \in C\left(x, c_{\lambda}\right)\right\}$ are the times at which individuals in $C\left(x, c_{\lambda}\right)$ meet the barrier hit $\Gamma^{\left(x, c_{\lambda}\right)}$ and $\mathcal{F}_{C\left(x, c_{\lambda}\right)}$ $(x \geqslant 0)$ is the natural filtration generated by ancestral and spatial paths receding from individuals at the moment that they hit $\Gamma^{\left(x, c_{\lambda}\right)}$ then given $\mathcal{F}_{C\left(x, c_{\lambda}\right)}$ each of the trees relative to and rooted at the space time points

$$
\left\{\left(\Xi_{u}\left(\sigma_{u}\right), \sigma_{u}\right) \in \mathbb{R} \times \mathbb{R}^{+}: u \in C\left(x, c_{\lambda}\right)\right\}
$$

are independent copies of the original branching process.

For further information on general stopping lines and properties, one should consult [14,27]. It should also be mentioned that for superdiffusions and branching Markov diffusions similar constructions to the one above are known as exit measures; see [19].

From the afore mentioned references, it is known that when $\lambda \in[0, \underline{\lambda})$ and $z>0$ and $\Phi_{c_{\lambda}}$ is any travelling wave at speed $c_{\lambda}$,

$$
M_{x}\left(z, c_{\lambda}\right):=\prod_{u \in C\left(x, c_{\lambda}\right)} \Phi_{c_{\lambda}}\left(z+\Xi_{u}\left(\sigma_{u}\right)+c_{\lambda} \sigma_{u}\right)=\Phi_{c_{\lambda}}(z+x)^{\left|C\left(x, c_{\lambda}\right)\right|}
$$

is a $P$-martingale with respect to $\left\{\mathcal{F}_{C\left(x, c_{\lambda}\right)}: x \geqslant 0\right\}$ having expectation $\Phi_{c_{\lambda}}(z)$ that converges almost surely and in mean (due to boundedness). It follows that

$$
\lim _{x \uparrow \infty}-\left|C\left(x, c_{\lambda}\right)\right| \log \Phi_{c_{\lambda}}(x)
$$

exists and the limit is strictly positive with positive probability.

Define for $x>0$

$$
W_{C\left(x, c_{\lambda}\right)}(\lambda)=\sum_{u \in C\left(x, c_{\lambda}\right)} e^{-\lambda\left(\Xi_{u}\left(\sigma_{u}\right)+c_{\lambda} \sigma_{u}\right)}=e^{-\lambda x}\left|C\left(x, c_{\lambda}\right)\right| .
$$

Theorem 8. The sequence $\left\{W_{C\left(x, c_{\lambda}\right)}(\lambda): x \geqslant 0\right\}$ is a P-martingale with respect to filtration $\left\{\mathcal{F}_{C\left(x, c_{\lambda}\right)}: x \geqslant 0\right\}$ that converges almost surely and in $L^{1}(P)$ to $W(\lambda)$ when $|\lambda| \in[0, \underline{\lambda})$ and $E\left(X \log ^{+} X\right)<\infty$.

Proof. Let

$$
C_{t}\left(x, c_{\lambda}\right)=\left\{u \in C\left(x, c_{\lambda}\right): \sigma_{u} \leqslant t\right\}
$$

be the set of individuals who reached $C\left(x, c_{\lambda}\right)$ up to time $t$ and

$$
A_{t}\left(C\left(x, c_{\lambda}\right)\right)=\left\{u \in N_{t}: v \notin C_{t}\left(x, c_{\lambda}\right) \forall v \leqslant u\right\}
$$


be the set of particles in $N_{t}$ whose ancestral lines have not yet met $C\left(x, c_{\lambda}\right)$ and define

$$
W_{t \wedge C\left(x, c_{\lambda}\right)}(\lambda)=\sum_{u \in A_{t}\left(C\left(x, c_{\lambda}\right)\right)} e^{-\lambda\left(\Xi_{u}(t)+c_{\lambda} t\right)}+e^{-\lambda x}\left|C_{t}\left(x, c_{\lambda}\right)\right| .
$$

By decomposing members of $N_{t}$ in accordance with their ancestors (if at all) in $C_{t}\left(x, c_{\lambda}\right)$, much as in (14), a straightforward calculation shows that

$$
E\left(W_{t}(\lambda) \mid \mathcal{F}_{C\left(x, c_{\lambda}\right)}\right)=W_{t \wedge C\left(x, c_{\lambda}\right)}(\lambda) .
$$

As $C\left(x, c_{\lambda}\right)$ is a dissecting stopping line,

$$
\lim _{t \uparrow \infty}\left|A_{t}\left(C\left(x, c_{\lambda}\right)\right)\right|=0 \quad \text { and } \quad \lim _{t \uparrow \infty}\left|C\left(x, c_{\lambda}\right) \backslash C_{t}\left(x, c_{\lambda}\right)\right|=0 \quad \text { a.s. }
$$

When $\lambda \in[0, \underline{\lambda})$ and $E\left(X \log ^{+} X\right)<\infty, W_{t}(\lambda)$ has an $L^{1}(P)$ limit equal to $W(\lambda)$ and hence $E\left(W_{t}(\lambda) \mid \mathcal{F}_{C\left(x, c_{\lambda}\right)}\right)$ has an $L^{1}(P)$ limit equal to $E\left(W(\lambda) \mid \mathcal{F}_{C\left(x, c_{\lambda}\right)}\right)$. With the previous remarks we now have

$$
\lim _{t \uparrow \infty} E\left(W_{t}(\lambda) \mid \mathcal{F}_{C\left(x, c_{\lambda}\right)}\right)=E\left(W(\lambda) \mid \mathcal{F}_{C\left(x, c_{\lambda}\right)}\right)=W_{C\left(x, c_{\lambda}\right)}(\lambda)
$$

almost surely showing that $W_{C\left(x, c_{\lambda}\right)}(\lambda)$ is an $\mathcal{F}_{C\left(x, c_{\lambda}\right)}$-martingale.

As the sequence $C\left(x, c_{\lambda}\right)$ is tending to infinity, then $\lim _{x \uparrow \infty} A_{t}\left(C\left(x, c_{\lambda}\right)\right)=N_{t}$ and $\lim _{x \uparrow \infty}\left|C_{t}\left(x, c_{\lambda}\right)\right|=0$ almost surely. Taking the limit in (19) with respect to $x$ instead thus gives us,

$$
E\left(W_{t}(\lambda) \mid \mathcal{F}_{\infty}\right)=W_{t}(\lambda)
$$

for all $t>0$ where $\mathcal{F}_{\infty}=\sigma\left(\bigcup_{x \geqslant 0} \mathcal{F}_{C\left(x, c_{\lambda}\right)}\right)$. This implies that $W_{t}(\lambda)$ is $\mathcal{F}_{\infty}$-measurable for each $t>0$ and thus so is its limit $W(\lambda)$. In conclusion $\lim _{x \uparrow \infty} W_{C\left(x, c_{\lambda}\right)}(\lambda)=W(\lambda)$. The theorem is proved.

The theorem also confirms that $\lambda$ is the Malthusian parameter of the branching process $\left\{\left|C\left(x, c_{\lambda}\right)\right|: x \geqslant 0\right\}$.

\subsection{Asymptotics and uniqueness at supercriticality}

Assume now the conditions of Theorem 8. Since we have shown that $W(\lambda)$ strictly positive with positive probability, we now have two sequences of (Seneta-Heyde) norming constants for the branching process $\left\{\left|C\left(x, c_{\lambda}\right)\right|: x \geqslant 0\right\}$. Consequently, these two norming sequences must be asymptotically equivalent on the event $\{W(\lambda)>0\}$. That is to say,

$$
\lim _{x \uparrow \infty} \frac{-\log \Phi_{c_{\lambda}}(x)}{e^{-\lambda x}}=\lim _{x \uparrow \infty} \frac{1-\Phi_{c_{\lambda}}(x)}{e^{-\lambda x}}=k
$$

where the second equality follows since $\Phi_{c_{\lambda}}(\infty)=1$ and $k$ is a positive constant. We have thus constructed an alternative proof of the asymptotic (4). Uniqueness (up to a multiplicative constant in the argument) is now immediate since

$$
\begin{aligned}
\Phi_{c_{\lambda}}(z) & =E\left(\lim _{x \uparrow \infty} M_{x}\left(z, c_{\lambda}\right)\right)=E\left(\lim _{x \uparrow \infty} \exp \left\{-e^{-\lambda(z+x)}\left|C\left(x, c_{\lambda}\right)\right| \frac{-\log \Phi_{c_{\lambda}}(z+x)}{e^{-\lambda(z+x)}}\right\}\right) \\
& =E\left(\exp \left\{-k e^{-\lambda z} W(\lambda)\right\}\right) .
\end{aligned}
$$

\section{Branching Brownian motion with a barrier}

Define the space-time barrier

$$
\Gamma^{(-x, \lambda)}:=\left\{(y, t) \in \mathbb{R} \times \mathbb{R}^{+}: y+\lambda t=-x\right\} .
$$


Suppose for each $t>0$ in the branching Brownian motion we define a subset of $N_{t}$, say $\widetilde{N}_{t}$, consisting of all individuals alive at time $t$ having ancestry (including themselves) whose spatial paths have not met $\Gamma^{(-x, \lambda)}$ by time $t$. The surviving individuals and their spatial paths up to and including the moment they meet the barrier $\Gamma^{(-x, \lambda)}$ we refer to as branching Brownian motion with a barrier. It is the 'branching process generalization' of a single Brownian motion killed at $\Gamma^{(-x, \lambda)}$.

Define

$$
V_{t}^{x}(\lambda)=\sum_{u \in \tilde{N}_{t}} \frac{x+\Xi_{u}(t)+\lambda t}{x} e^{-\lambda\left(\Xi_{u}(t)+c_{\lambda} t\right)} .
$$

Theorem 9. The sequence $\left\{V_{t}^{x}(\lambda): t \geqslant 0\right\}$ is a mean $1 P$-martingale with respect to $\left\{\mathcal{F}_{t}: t \geqslant 0\right\}$ for all $\lambda \in \mathbb{R}$.

Proof. Using notation and concepts from Section 3 consider the stopping line

$$
\mathcal{L}(t)=\left\{u \in C(-x, \lambda): \sigma_{u} \leqslant t\right\} \cup \tilde{N}_{t} .
$$

Let $\mathcal{F}_{\mathcal{L}(t)}$ be the natural filtration generated by the ancestral and spatial paths receding from individuals at the moment they enter $\mathcal{L}(t)$. A straightforward computation using the Strong Markov Branching Property shows that for all $0 \leqslant s \leqslant t$

$$
E\left(\partial W_{t}(\lambda)+x W_{t}(\lambda) \mid \mathcal{F}_{\mathcal{L}(t)}\right)=x V_{t}^{x}(\lambda)
$$

thus showing that $E\left(V_{t}^{x}(\lambda)\right)=1$ for all $x, t \geqslant 0$. Using again the Strong Markov Branching Property, we have for $0 \leqslant s \leqslant t$,

$$
E\left(V_{t}^{x}(\lambda) \mid \mathcal{F}_{s}\right)=\sum_{u \in \widetilde{N}_{s}} \frac{x+\Xi_{u}(s)+\lambda s}{x} e^{-\lambda\left(\Xi_{u}(s)+c_{\lambda} s\right)} E\left(V_{t-s}^{\left(x+\Xi_{u}(s)+\lambda s\right)}(\lambda, u) \mid \mathcal{F}_{s}\right),
$$

where $V_{t-s}^{(\cdot)}(\lambda, u)$ are independent copies of $V_{t-s}^{(\cdot)}(\lambda)$. Since $E V_{t}^{x}(\lambda)=1$ for all $x, t>0$ the proof is complete.

The following corollary shows why this martingale has significance with respect to establishing the convergence of the derivative martingale.

Corollary 10. Suppose that $\lambda \geqslant \underline{\lambda}$. Then $\partial W(\lambda)=\lim _{t \uparrow \infty} \partial W_{t}(\lambda)$ exists almost surely in $[0, \infty)$. Further, $P(\partial W(\lambda)=0)=0$ or 1 .

Proof. Note that $V_{t}^{x}(\lambda)$ is always positive and therefore has a limit almost surely. Further, we can identify $x V_{t}^{x}(\lambda)$ as contributing to the positive part of the martingale $\partial W_{t}(\lambda)+x W_{t}(\lambda)$ and thus serves as an approximation to it. In fact, on the event (which we shall call $\gamma^{(-x, \lambda)}$ ) that the branching Brownian motion remains entirely to the right of $\Gamma^{(-x, \lambda)}$, the truncated process and the original process are the same. It follows that on $\gamma^{(-x, \lambda)}$ the $\lim _{t \uparrow \infty} \partial W_{t}(\lambda)+x W_{t}(\lambda)$ exists and equals $\lim _{t \uparrow \infty} x V_{t}^{x}(\lambda) \geqslant 0$. Since for $\lambda \geqslant \underline{\lambda}$ the martingale limit $W(\lambda) \equiv 0$ it follows that on $\gamma^{(-x, \lambda)}$ we have

$$
\lim _{t \uparrow \infty} x V_{t}^{x}(\lambda)=\lim _{t \uparrow \infty} \partial W_{t}(\lambda) .
$$

Recall that $\lim _{t \uparrow \infty} L_{t}+\underline{c} t=\infty$ and hence $\inf _{t \geqslant 0}\left\{L_{t}+\lambda t\right\}>-\infty$ almost surely for all $\lambda \geqslant \underline{\lambda}$. Consequently

$$
P\left(\gamma^{(-x, \lambda)}\right)=P\left(\inf _{t \geqslant 0}\left\{L_{t}+\lambda t\right\}>-x\right) \uparrow 1 \quad \text { as } x \uparrow \infty .
$$

Thus we have established the existence of an almost sure limit for the derivative martingale with all its mass in $[0, \infty)$. Note also from (20) that when $\lambda \geqslant \underline{\lambda}, \partial W_{t}(\lambda)$ cannot converge in mean even when it converges almost surely. 
The following argument, modified from [26], shows that in fact in this regime of $\lambda, \partial W(\lambda)=\lim _{t \uparrow} \partial W_{t}(\lambda)$ is either strictly positive or zero with probability one. Let $q=P(\partial W(\underline{\lambda})=0)$. It is very easy if not a little messy (see, for example, [26]) to decompose $\partial W_{t}(\underline{\lambda})$ into contributions derived from the population at time $s \in(0, t)$ as was done in (14). We have

$$
\partial W_{t}(\underline{\lambda}) \stackrel{d}{=} \sum_{u \in N_{s}} e^{-\underline{\lambda}\left(\Xi_{u}(s)+\underline{\lambda} s\right)} \partial W_{t-s}(\underline{\lambda}, u)+\sum_{u \in N_{s}}\left(\Xi_{u}(s)+\underline{\lambda} s\right) e^{-\underline{\lambda}\left(\Xi_{u}(s)+\underline{\lambda} s\right)} W_{t-s}(\underline{\lambda}, u),
$$

where, for each $u \in N_{s}, \partial W_{t-s}(\underline{\lambda}, u)$ and $W_{t-s}(\underline{\lambda}, u)$ are independent copies of $\partial W_{t-s}(\underline{\lambda})$ and $W_{t-s}(\underline{\lambda})$ respectively. Using obvious notation, as $t$ tends to infinity we thus recover the identity

$$
\partial W(\underline{\lambda}) \stackrel{d}{=} \sum_{u \in N_{s}} e^{-\underline{\lambda}\left(\Xi_{u}(s)+\underline{\lambda} s\right)} \partial W(\underline{\lambda}, u)
$$

which holds for all $s>0$. It is immediate from this decomposition that

$$
q=E\left(q^{Z_{t}(\mathbb{R})}\right) \text {. }
$$

By the Markov property, it follows that $\left\{q^{Z_{t}(\mathbb{R})}: t \geqslant 0\right\}$ is a positive bounded martingale. Unless $q=1$ this martingale will tend to zero and then its $L^{1}(P)$ convergence forces $q=0$.

Remark 11. The idea of the approximation in the proof of the above corollary stems from a similar construction used in [26] where the martingale $\partial W_{t}(\lambda)+x W_{t}(\lambda)$ is stopped at the first time the left most particle hits the space time barrier $\Gamma^{(-x, \lambda)}$. Effectively we have replaced Harris' use of stopping times by stopping lines.

Remark 12. In the previous discussion it is quite important that $\lambda \geqslant \underline{\lambda}$. When $0<\lambda<\underline{\lambda}$, similar calculations to those found in Remark 7 show that $P\left(\gamma^{(-x, \lambda)}\right)=0$ for all $x>0$. The consequence of this is that $x V_{t}^{x}(\lambda)$ no longer serves its purpose as a good approximation to $\partial W_{t}(\lambda)$.

It is now clear that the positivity of $\partial W(\lambda)$ can be established from the positivity of the limit of $V_{t}^{x}(\lambda)$. This becomes the purpose of the next three sections.

\section{Brownian motion and Bessel-3 processes}

Let us now quote some results that show the intimate relationship between Brownian motion and Bessel-3 processes that will be of use later in this paper. Our main references are $[37,45,41,4]$.

Recall it was assumed that with respect to the law $\mathbb{P}, B=\left\{B_{t}: t \geqslant 0\right\}$ is a standard Brownian motion started at 0 and if $\left\{\mathcal{H}_{t}: t \geqslant 0\right\}$ is a filtration with respect to which $B$ is adapted then write $\mathbb{P}_{t}$ for the restriction of $\mathbb{P}$ to $\mathcal{H}_{t}$. Define

$$
\rho^{\lambda}(x+B)=\inf \left\{t>0: x+B_{t}+\lambda t=0\right\},
$$

the first time that $B$ meets the barrier

$$
\Gamma^{(-x, \lambda)}:=\left\{(y, t) \in \mathbb{R} \times \mathbb{R}^{+}: y+\lambda t=-x\right\} .
$$

Now say $\mathbb{Q}^{(-x, \lambda)}$ is the law under which the process $\left\{x+B_{t}+\lambda t: t \geqslant 0\right\}$ is a standard Bessel-3 process started at $x>0$ and write

$$
\Lambda_{t}^{(-x, \lambda)}(B)=\frac{x+B_{t}+\lambda t}{x} e^{-\lambda\left(B_{t}+\lambda t / 2\right)} \mathbf{1}_{\left(\rho^{\lambda}(x+B)>t\right)} .
$$


It can be easily checked from the fact that $\mathbb{Q}^{(-x, 0)}$ is also the law of a Brownian motion started at 0 and conditioned not to enter the half-line $(-\infty,-x]$ together with the Cameron-Martin-Girsanov theorem that

$$
\frac{d \mathbb{Q}_{t}^{(-x, \lambda)}}{d \mathbb{P}_{t}}(B)=\Lambda_{t}^{(-x, \lambda)}(B) .
$$

Indeed $\mathbb{Q}^{(-x, \lambda)}$ is also the law of a Brownian motion started at 0 and conditioned not to meet the space-time barrier $\Gamma^{(-x, \lambda)}$.

\section{Change of measure inducing a conditioned spine}

Having now seen that the density $\Lambda_{t}^{(-x, \lambda)}(B)$ can be used to condition a Brownian motion not to hit the space time barrier $\Gamma^{(-x, \lambda)}$ (thus producing a Bessel-3 process with drift) it is natural to ask what the effect on the branching Brownian motion is when using $V_{t}^{x}(\lambda)$ as a change of measure.

Using the same notation and conventions as in Section 2 consider now a new probability measure $\Pi_{t}^{*}$ on $\left(\widetilde{\mathcal{T}}, \widetilde{\mathcal{F}}_{t}\right)$ such that

$$
\begin{aligned}
d \Pi_{t}^{*}(\tau, B, \eta, \xi)= & e^{-\beta m t} \Lambda_{t}^{(-x, \lambda)}(\Xi) d P_{t}^{*}(\tau, B, \eta, \xi)=\Lambda_{t}^{(-x, \lambda)}(\Xi) e^{-\beta m t}(m+1)^{n_{t}} \\
& \times \prod_{v<\xi_{n_{t}}}\left(\frac{X_{v}+1}{m+1}\right) \frac{1}{X_{v}+1} d P_{t}^{*}(\tau, B, \eta, \xi) \\
= & d \mathbb{Q}_{t}^{(-x, \lambda)}(\Xi) d \mathbb{L}_{t}^{(\beta(m+1))}(n) \prod_{v<\xi_{n t}}\left(\frac{X_{v}+1}{m+1}\right) p_{X_{v}} \frac{1}{X_{v}+1} \prod_{j=1}^{X_{v}} d P_{t-v_{v}}\left((\tau, B, \eta)_{j}^{v}\right)
\end{aligned}
$$

thus inducing a probability measure $\Pi^{*}$ on $(\widetilde{\mathcal{T}}, \widetilde{\mathcal{F}})$. The fact that $\Pi_{t}^{*}$ is a probability measure can again be confirmed with the help of (7). Further, with calculations much along the lines of those in Section 2.1 one can check by marginalizing $\Pi_{t}^{*}$ to $\left(\mathcal{T}, \mathcal{F}_{t}\right)$ we find again a probability measure $\Pi_{t}$ satisfying

$$
\frac{d \Pi_{t}}{d P_{t}}=V_{t}^{x}(\lambda)
$$

which in turn induces a measure on $(\mathcal{T}, \mathcal{F})$ which we shall call $\Pi$. The effect of this change of measure on $\left\{Z_{t}: t \geqslant 0\right\}$ corresponds to the law of a non-homogeneous branching process with distinguished and randomized spine having the following properties:

(i) the diffusion along the spine is such that $\{x+\Xi(t)+\lambda t: t \geqslant 0\}$ is a Bessel-3 process on $(0, \infty)$ started at $x$ (that is to say the diffusion along the spine, $\Xi$, moves away from the barrier $\Gamma^{(-x, \lambda)}$ as a Bessel-3 processes and therefore never meets it),

(ii) the points of fission along the spine form a Poisson process with accelerated rate $(m+1) \beta$,

(iii) the distribution of offspring numbers at each point of fission on the spine has tilted measure $\left(\tilde{p}_{k}=(k+\right.$ 1) $\left.p_{k} /(m+1): k \geqslant 0\right)$

(iv) the spine is chosen randomly so that at each fission point, the next individual to represent the spine is chosen with uniform probability from the offspring of the current representative,

(v) individuals which do not carry the spine evolve as $P$-branching Brownian motions. 


\section{Proof of Theorem 3}

Again pursuing the same line as Lyons [35] and Lyons et al. [36] we define for any $x>0, V^{x}(\lambda)=$ $\lim \sup _{t \uparrow \infty} V_{t}^{x}(\lambda)$ (which is also equal to $\lim _{t \uparrow \infty} V_{t}^{x}(\lambda) P$-a.s.) and check whether this limit has any $P$-mass away from zero by using again the fundamental measure theoretic result

$$
\begin{aligned}
V^{x}(\lambda)=\infty \Pi \text {-a.s. } & \Longleftrightarrow V^{x}(\lambda)=0 P \text {-a.s. } \\
V^{x}(\lambda)<\infty \Pi \text {-a.s. } & \Longleftrightarrow \int V^{x}(\lambda) d P=1 .
\end{aligned}
$$

Theorem 13. For $x>0$, the almost sure limit $V^{x}(\lambda)$ has the following properties

(i) If $\lambda>\underline{\lambda}$ then $V^{x}(\lambda)=0 P$-a.s.

(ii) If $\lambda=\underline{\lambda}$ then $V^{x}(\lambda)=0$ P-a.s. or is an $L^{1}(P)$-limit accordingly as $E\left(X\left(\log ^{+} X\right)^{2-\delta}\right)=\infty$ or $E\left(X\left(\log ^{+} X\right)^{2+\delta}\right)<\infty$ for some $\delta>0$.

(iii) If $\lambda \in[0, \underline{\lambda})$ then $V^{x}(\lambda)=0 P$-a.s. or is an $L^{1}(P)$-limit accordingly as $E\left(X \log ^{+} X\right)=\infty$ or $E\left(X \log ^{+} X\right)<$ $\infty$.

Remark 14. Now note the proof of Theorem 3 is a direct result of parts (i)-(ii) of the above theorem and Corollary 10 .

Before proceeding to the proof of Theorem 13 we need to note a few things about the behaviour of Bessel-3 processes and also the asymptotics of a sequence $\left\{X_{n}: n \geqslant 0\right\}$ of independent copies of $X$ representing the numbers of offspring of along the spine at each point of fission.

Firstly, from Theorem 3.2 of [42] it is easy to show that for any $\varepsilon>0$, the path of a Bessel-3 process becomes bounded by the curves $t^{1 / 2+\varepsilon}$ and $t^{1 / 2-\varepsilon}$ for all sufficiently large times $\mathbb{Q}^{(-x, 0)}$-almost surely. Secondly a simple calculation shows that when $q>0 E\left(X\left(\log ^{+} X\right)^{q}\right)$ is (in)finite if and only if

$$
\sum_{n \geqslant 1} \tilde{\operatorname{Pr}}\left(\log X>c n^{1 / q}\right)
$$

is (in)finite for any $c>0$ where again $\widetilde{\operatorname{Pr}}$ is the probability measure under which $X$ has distribution $\left(\tilde{p}_{k}: k \geqslant 0\right)$. Consequently, by the Borel-Cantelli lemma,

$$
\limsup _{n \uparrow \infty} n^{-1 / q} \log X_{n}
$$

is (infinite) zero according whether the given moment is (in)finite.

Proof of Theorem 13. (i) Suppose that $\lambda>\underline{\lambda}$. By construction we have the lower bound

$$
V_{t}^{x}(\lambda) \geqslant \frac{x+\Xi(t)+\lambda t}{x} e^{-\lambda\left(\Xi(t)+c_{\lambda} t\right)}
$$

Recall that under $\Pi^{*},\{x+\Xi(t)+\lambda t\}_{t>0}$ is a Bessel-3 process on $(0, \infty)$ started at $x$. It is known (cf. Theorem 3.2 of [42]) that this process eventually grows no faster than $t^{1 / 2+\varepsilon}$ for any $\varepsilon>0$ and since for $\lambda>\underline{\lambda}, c_{\lambda}<\lambda$ it becomes clear from (28) that $V^{x}(\lambda)=\infty \Pi$-almost surely and hence $V^{x}(\lambda)$ is identically zero $P$-almost surely by (26).

(ii) Now suppose that $\lambda=\underline{\lambda}$ (so that $c_{\lambda}=\underline{c}=\underline{\lambda}$ ) and $E\left(X\left(\log ^{+} X\right)^{2-\delta}\right)=\infty$ for some $\delta>0$. Without loss of generality we can assume that $\delta$ is small. We have the lower bound

$$
V_{\nu_{\xi_{k}}}^{x}(\underline{\lambda}) \geqslant X_{\xi_{k}} \frac{x+\Xi\left(\nu_{\xi_{k}}\right)+\underline{\lambda} \nu_{\xi_{k}}}{x} e^{-\underline{\lambda}\left(\Xi\left(\nu_{\xi_{k}}\right)+\lambda v_{\xi_{k}}\right)}
$$


where $\xi$ is the spine. By the law of large numbers, $v_{k} \sim[\beta(m+1)]^{-1} k$. We deduce that $\left(x+\Xi\left(\nu_{\xi_{k}}\right)+\underline{\lambda} \nu_{\xi_{k}}\right)$ eventually grows no faster than $\mathrm{O}\left(k^{1 / 2+\varepsilon}\right)$ for any $\varepsilon>0$. Hence by choosing $\varepsilon$ sufficiently small, (29) shows that with the given moment condition, the term $X_{\xi_{k}}$ dominates the behaviour of the Bessel-3 process and $V^{x}(\underline{\lambda})=\infty$ $\Pi$-almost surely. Thus the result follows as before.

Suppose now that $\lambda=\underline{\lambda}$ (so that $c_{\lambda}=\underline{c}=\underline{\lambda}$ ) and $E\left(X\left(\log ^{+} X\right)^{2+\delta}\right)<\infty$ for some $\delta>0$. Let $\mathcal{G}$ be the sigmaalgebra generated by the diffusion of the spine $\xi$, the Poisson process $n$ representing the times of reproduction and $\left\{X_{n_{t}}: t \geqslant 0\right\}$. We have

$$
\begin{aligned}
E_{\Pi^{*}}\left(V_{t}^{x}(\underline{\lambda}) \mid \mathcal{G}\right)= & \sum_{i=1}^{n_{t}} X_{\xi_{i-1}} \frac{x+\Xi\left(\nu_{\xi_{i-1}}\right)+\underline{\lambda} \nu_{\xi_{i-1}}}{x} e^{-\underline{\lambda}\left(\Xi\left(\nu_{\xi_{i-1}}\right)+\underline{\lambda} v_{\xi_{i-1}}\right)} \\
& +\frac{x+\Xi(t)+\underline{\lambda} t}{x} e^{-\underline{\lambda}(\Xi(t)+\underline{\lambda} t)} .
\end{aligned}
$$

Taking advantage of the fact that a Bessel-3 process eventually grows no slower than $t^{1 / 2-\varepsilon}$ for any $\varepsilon>0$, the law of large numbers applied to $n$ and the sequence $\left(v_{i}: i \geqslant 1\right)$ plus the given moment condition implies that

$$
\underset{t \uparrow \infty}{\limsup } E_{\Pi^{*}}\left(V_{t}^{x}(\underline{\lambda}) \mid \mathcal{G}\right)<\infty \quad \Pi^{*} \text {-a.s. }
$$

Fatou's lemma implies that $\liminf _{t \uparrow \infty} V_{t}^{x}(\underline{\lambda})$ is also finite $\Pi^{*}$-almost surely. The Radon-Nikodym derivative (25) tells us that $V_{t}^{x}(\underline{\lambda})^{-1}$ is $\Pi$-martingale and therefore has a limit $\Pi$-almost surely. It follows now that $\lim \sup _{t \uparrow \infty} V_{t}^{x}(\underline{\lambda})=\liminf _{t \uparrow \infty} V_{t}^{x}(\underline{\lambda})<\infty \Pi$-almost surely. From (27) we thus conclude that $V^{x}(\underline{\lambda})$ is an $L^{1}(P)$ limit.

(iii) Let $\lambda \in[0, \underline{\lambda})$ and $E\left(X \log ^{+} X\right)=\infty$. Note now that $c_{\lambda}>\lambda$. We have

$$
V_{v_{\xi_{k}}}^{x}(\lambda) \geqslant X_{\xi_{k}} \frac{x+\Xi\left(\nu_{\xi_{k}}\right)+\lambda v_{\xi_{k}}}{x} e^{-\lambda\left(\Xi\left(v_{\xi_{k}}\right)+\lambda v_{k}\right)} e^{-\lambda\left(c_{\lambda}-\lambda\right) v_{\xi_{k}}} .
$$

Following similar reasoning to parts (i) and (ii) it can be seen that the leading order in the exponent can be compensated by the term $X_{\xi_{k}}$ to show again that $V^{x}(\lambda)=\infty \Pi$-almost surely.

When $\lambda \in[0, \underline{\lambda})$ and $E\left(X \log ^{+} X\right)<\infty$ we can make again the familiar decomposition

$$
\begin{aligned}
E_{\Pi^{*}}\left(V_{t}^{x}(\lambda) \mid \mathcal{G}\right)= & \sum_{i=1}^{n_{t}} X_{\xi_{i-1}} \frac{x+\Xi\left(\nu_{\xi_{i-1}}\right)+\lambda v_{\xi_{i-1}}}{x} e^{-\lambda\left(\Xi\left(v_{\xi_{i-1}}\right)+\lambda v_{i}\right)} e^{-\lambda\left(c_{\lambda}-\lambda\right) v_{\xi_{i-1}}} \\
& +\frac{x+\Xi(t)+\lambda t}{x} e^{-\lambda(\Xi(t)+\lambda t)} e^{-\lambda\left(c_{\lambda}-\lambda\right) t}
\end{aligned}
$$

and similar reasoning to part (ii) completes the proof.

\section{Existence, asymptotics and uniqueness at criticality}

We are now ready to show existence and uniqueness of travelling wave solutions to the K-P-P equation at the critical wave speed. To this end we assume that $E\left(X\left(\log ^{+} X\right)^{2+\delta}\right)<\infty$ for some $\delta>0$. Now that we are in possession of the strictly positive limit $\partial W(\underline{\lambda})$ we can use similar martingale tricks to those used in Sections 2 and 3 to finish up. 


\subsection{Existence at criticality}

The identity (23) given in the proof of Corollary 10 can be also expressed in terms of Laplace transforms. That is to say, if $\Phi(x):=E\left(\exp \left\{-e^{-\underline{\lambda} x} \partial W(\underline{\lambda})\right\}\right)$, then $\Phi$ satisfies the functional equation

$$
\Phi(x)=E\left[\prod_{u \in N_{s}} \Phi\left(x+\Xi_{u}(s)+\underline{\lambda} s\right)\right]
$$

for all $s>0$. Once again we appeal to the fact that $\Phi$ solves the functional equation (30) if and only if is also solves the travelling wave equation (2); cf. [38,13]. Boundedness, monotonicity and the limits of $\Phi$ at $\pm \infty$ are all easily verified as before. Hence we have shown existence.

\subsection{Asymptotics at criticality}

Consider the barrier $\Gamma^{(z, \underline{\lambda})}$ where $z>0$. By arresting lines of decent the first time they hit this barrier we again produce a sequence of stopping lines $\left\{C^{(z, \underline{\lambda})}: z \geqslant 0\right\}$ which are dissecting and tending to infinity (refer to Section 3). Now let $\left\{\sigma_{u}: u \in C^{(z, \underline{\lambda})}\right\}$ be the times at which individuals meet the barrier $\Gamma^{(z, \underline{\lambda})}$. From [14,39], it is known that for any travelling wave at the critical speed, $\Phi_{\underline{c}}$,

$$
\prod_{u \in C^{(z, \underline{\lambda})}} \Phi_{\underline{c}}\left(\Xi_{u}\left(\sigma_{u}\right)+\underline{\lambda} \sigma_{u}\right)=\Phi_{\underline{c}}(z)^{\left|C^{(z, \underline{\lambda})}\right|}
$$

is a martingale with a limit almost surely and in $L^{1}(P)$ with mean $\Phi_{\underline{c}}(0)$.

Suppose we turn our attention to the branching Brownian motion with a killing barrier at $\Gamma^{(-x, \underline{\lambda})}$ (recall Section 4). Define $\widetilde{C}^{(z, \underline{\lambda})}$ as the set of individuals in the process with killing at $\Gamma^{(-x, \underline{\lambda})}$ that are stopped at the barrier $\Gamma^{(z, \underline{\lambda})}$ for $z>0$. For the sake of clarity we remark that $\widetilde{C}^{(z, \underline{\lambda})}$ consists of particles whose lines of descent (including themselves) have spatial paths that have met the barrier $\Gamma^{(z, \underline{\lambda})}$ before meeting the barrier $\Gamma^{(-x, \underline{\lambda})}$. Recall that on the event $\gamma^{(-x, \underline{\lambda})}$ the branching Brownian motion and the branching Brownian motion with the barrier $\Gamma^{(-x, \underline{\lambda})}$ are one and the same $\left(\gamma^{(-x, \underline{\lambda})}\right.$ corresponds to the event that no particle ever meets $\left.\Gamma^{(-x, \underline{\lambda})}\right)$. Thus we can say that

$$
\lim _{z \uparrow \infty}-\left|\widetilde{C}^{(z, \underline{\lambda})}\right| \log \Phi_{\underline{c}}(z)
$$

exists almost surely and is in $[0, \infty)$ on $\gamma^{(-x, \underline{\lambda})}$ because of the same statement being true for $\lim _{z \uparrow \infty}-\left|C^{(z, \underline{\lambda})}\right| \times$ $\log \Phi_{\underline{c}}(z)$. Further we claim that this limit is non-trivial in the sense that for all sufficiently large $x>0$, $\lim _{z \uparrow \infty}-\left|\widetilde{C}^{(z, \underline{\lambda})}\right| \log \Phi_{\underline{c}}(z)$ is valued in $(0, \infty)$ with positive probability on $\gamma^{(-x, \underline{\lambda})}$. To see this, suppose that the contrary were true. This is equivalent to supposing that the $\lim _{z \uparrow \infty} \Phi_{\underline{c}}(z)^{\left|C^{(z, \underline{\lambda})}\right|}=1$ on $\gamma^{(-x, \underline{\lambda})}$ for all $x>0$; that is to say that the given limit is equal to one everywhere except on $\left\{L_{t}+\underline{\lambda} t \rightarrow-\infty\right\}$ which, as we have seen in Section 4, is a $P$-null set. This provides us with a contradiction since otherwise we are forced by $L^{1}(P)$ martingale convergence to conclude that $\Phi_{\underline{c}}(0)=1$ and hence by monotonicity of $\Phi_{\underline{c}}$ and the fact that $\Phi_{\underline{c}}(\cdot+y)$ is still a travelling wave for any $y \in \mathbb{R}$ it would follow that $\Phi_{\underline{c}} \equiv 1$. It therefore follows that (31) is strictly positive with positive probability on $\gamma^{(-x, \underline{\lambda})}$ for some and hence all sufficiently large $x>0$.

Our goal now is to look for another normalization of the sequence $\left\{\left|\widetilde{C}^{(z, \underline{\lambda})}\right|: z \geqslant 0\right\}$ which will supply us with the required asymptotic. To this end consider the process

$$
V_{\widetilde{C}^{(z, \underline{\lambda})}}^{x}:=\sum_{u \in \widetilde{C}^{(z, \underline{\lambda})}} \frac{x+\Xi_{u}\left(\sigma_{u}\right)+\underline{\lambda} \sigma_{u}}{x} e^{-\underline{\lambda}\left(\Xi_{u}\left(\sigma_{u}\right)+\underline{\lambda} \sigma_{u}\right)}=\frac{x+z}{x} e^{-\underline{\lambda} z}\left|\widetilde{C}^{(z, \underline{\lambda})}\right| .
$$

Using ideas similar to those in the proof of Theorem 8 we can prove the following theorem. 
Theorem 15. Let $\left\{\mathcal{F}_{\widetilde{C}^{(z, \lambda)}}: z \geqslant 0\right\}$ be the natural filtration describing everything in the truncated branching tree up

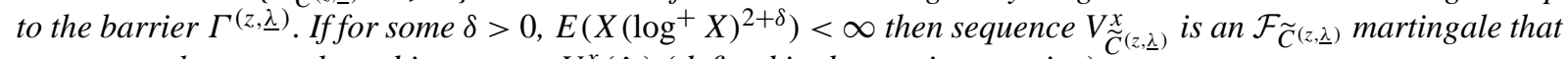
converges almost surely and in mean to $V^{x}(\underline{\lambda})$ (defined in the previous section).

Proof. For $t>0$, let

$$
\widetilde{C}_{t}^{(z, \underline{\lambda})}=\left\{u \in \widetilde{C}^{(z, \underline{\lambda})}: \sigma_{u} \leqslant t\right\}
$$

and

$$
A_{t}\left(\widetilde{C}^{(z, \underline{\lambda})}\right)=\left\{u \in \widetilde{N}_{t}: v \notin \widetilde{C}_{t}^{(z, \underline{\lambda})} \forall v \leqslant u\right\}
$$

and define

$$
V_{t \wedge \widetilde{C}^{(z, \underline{\lambda})}}^{x}=\sum_{u \in A_{t}\left(\widetilde{C}^{(z, \underline{\lambda})}\right)} \frac{x+\Xi_{u}(t)+\underline{\lambda} t}{x} e^{-\underline{\lambda}\left(\Xi_{u}(t)+\underline{\lambda} t\right)}+\frac{(x+z)}{x} e^{-\underline{\lambda} z}\left|\widetilde{C}_{t}^{(z, \underline{\lambda})}\right| .
$$

By decomposing members of $\widetilde{N}_{t}$ in accordance with their ancestors (if at all) in $\widetilde{C}^{(z, \underline{\lambda})}$, it is a straightforward calculation, similar to (14), to show that

$$
E\left(V_{t}^{x}(\underline{\lambda}) \mid \mathcal{F}_{\widetilde{C}^{(z, \underline{\lambda})}}\right)=V_{t \wedge \widetilde{C}^{(z, \underline{\lambda})}}^{x} .
$$

Since $\widetilde{C}^{(z, \underline{\lambda})}$ is a dissecting stopping line tending to infinity, $\lim _{t \uparrow \infty}\left|A_{t}\left(\widetilde{C}^{(z, \underline{\lambda})}\right)\right|=0$ and $\lim _{t \uparrow \infty} \widetilde{C}_{t}^{(z, \underline{\lambda})}=\widetilde{C}^{(z, \underline{\lambda})}$. From the proof of Theorem $3, V_{t}^{x}(\underline{\lambda})$ converges in mean to $V^{x}(\underline{\lambda})$ as $t$ tends to infinity we thus have

$$
\lim _{t \uparrow \infty} E\left(V_{t}^{x}(\underline{\lambda}) \mid \mathcal{F}_{\left.\widetilde{C}^{(z, \underline{\lambda}}\right)}\right)=E\left(V^{x}(\underline{\lambda}) \mid \mathcal{F}_{\left.\widetilde{C}^{(z, \underline{\lambda})}\right)}\right)=V_{\widetilde{C}^{(z, \underline{\lambda})}}^{x}
$$

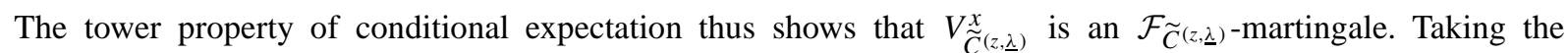
limit in (32) with respect to $z$ instead gives us similarly $E\left(V_{t}^{x}(\underline{\lambda}) \mid \tilde{\mathcal{F}}_{\infty}^{(z, \lambda)}\right)=V_{t}^{x}(\underline{\lambda})$ for all $t>0$ where now $\mathcal{F}_{\infty}=\sigma\left(\bigcup_{z \geqslant 0} \mathcal{F}_{\widetilde{C}^{(z, \underline{\lambda})}}\right)$. This implies that $V_{\underline{\lambda}}^{x}$ is $\mathcal{F}_{\infty}$-measurable and hence $V_{\left.\widetilde{C}^{(z, \underline{\lambda}}\right)}^{x}$ has limit $V^{x}(\underline{\lambda})$ as $z$ tends to infinity.

We can now prove the asymptotic we are after. Recall that for some $\delta>0, E\left(X\left(\log ^{+} X\right)^{2+\delta}\right)<\infty$. We have for each $x>0$,

$$
\lim _{z \uparrow \infty} \frac{-\log \Phi_{\underline{c}}(z)}{z e^{-\underline{\lambda} z}}=\lim _{z \uparrow \infty} \frac{(x+z)}{x z} \frac{-\left|\widetilde{C}^{(z, \underline{\lambda})}\right| \log \Phi_{\underline{\lambda}}(z)}{x^{-1}(x+z) e^{-\underline{\lambda} z}\left|\widetilde{C}^{(z, \underline{\lambda})}\right|}
$$

where the (non-stochastic) limit on the left hand side exists because it exists on the right hand side (at least on $\left\{V^{x}(\underline{\lambda})>0\right\}$ which is positive with positive probability). Note that this also implies that the limit on the right-hand side must also be a constant in $[0, \infty)$. This limiting constant may also be zero since $\lim _{z \uparrow \infty}-\left|\widetilde{C}^{(z, \underline{\lambda})}\right| \log \Phi_{\underline{\lambda}}(z)$ may also be zero valued. On the other hand, since this statement is valid for all $x>0$, and for $x$ sufficiently large we know that $\lim _{z \uparrow \infty}-\left|\widetilde{C}^{(z, \underline{\lambda})}\right| \log \Phi_{\underline{\lambda}}(z)$ is positive with positive probability on $\gamma^{(-x, \underline{\lambda})}$, we are forced to conclude that

$$
\lim _{z \uparrow \infty} \frac{-\log \Phi_{\underline{c}}(z)}{z e^{-\underline{\lambda} z}}=c
$$

for some constant $c \in(0, \infty)$ and hence since $\Phi_{\underline{\lambda}}(\infty)=1$ the asymptotic (3) is proved.

\subsection{Uniqueness at criticality}

The argument we now offer for uniqueness is essentially the same as in [26]. Solutions to (30) can also be used to construct $L^{1}(P)$-convergent multiplicative martingales of the form

$$
M_{t}(x):=\prod_{u \in N_{t}} \Phi_{\underline{c}}\left(x+\Xi_{u}(t)+\underline{\lambda} t\right) \quad \text { for } t>0 .
$$


Using the asymptotic behaviour of $\Phi_{\underline{c}}$, since $L_{t}+\underline{\lambda} t \rightarrow \infty$ a.s., we have that

$$
\begin{aligned}
-\log M_{t}(x) & \sim \sum_{u \in N_{t}}-\log \Phi_{\underline{c}}\left(x+\Xi_{u}(t)+\underline{\lambda} t\right) \sim \sum_{u \in N_{t}}\left[1-\Phi_{\underline{c}}\left(x+\Xi_{u}(t)+\underline{\lambda} t\right)\right] \\
& \sim k \sum_{u \in N_{t}}\left(x+\Xi_{u}(t)+\underline{\lambda} t\right) e^{-\underline{\lambda}\left(x+\Xi_{u}(t)+\underline{\lambda} t\right)}=k e^{-\underline{\lambda} x}\left(\partial W_{t}(\underline{\lambda})+x W_{t}(\underline{\lambda})\right)
\end{aligned}
$$

as $t$ tends to infinity where is a constant. Thus any solution to (2) at criticality must satisfy

$$
\Phi_{\underline{c}}(x)=E\left[\lim _{t \uparrow \infty} M_{t}(x)\right]=E\left(\exp \left\{-k e^{-\underline{\lambda} x} \partial W(\underline{\lambda})\right\}\right)
$$

(since $W(\underline{\lambda})=0$ almost surely) and therefore uniquenesses (modulo an additive constant in the argument) follows.

\section{Acknowledgements}

I thank Ross Pinsky, John Biggins and two anonymous referees for their valuable remarks and technical observations. In particular one of the referees was responsible for a number of non-trivial refinements to the arguments. Some of this work was carried out whilst visiting Amir Kabir University of Technology and the Tehran Teacher Training University in Iran. The author expresses his sincere thanks for their kind hospitality and support.

\section{References}

[1] D.G. Aronson, H.F. Weinberger, Nonlinear diffusions in population genetics, combustion and nerve propagation, in: J. Goldstein (Ed.), Partial Differential Equations and Related Topics, in: Lecture Notes in Math., vol. 446, Springer-Verlag, Berlin/New York, 1975.

[2] K. Athreya, P. Ney, Branching Processes, Springer-Verlag, Berlin, 1972.

[3] K. Athreya, Change of measures for Markov chains and the LlogL theorem for branching processes, Bernoulli 6 (1999) $323-338$.

[4] J. Bertoin, Splitting at the infimum and excursions in half-lines for random walks and Lévy processes, Stochastic Process. Appl. 42 (1993) 307-313.

[5] M. Bachmann, Limit theorems for the minimal position in a branching random walk with independent logconcave displacements, Adv. in Appl. Probab. 32 (2000) 159-176.

[6] J.D. Biggins, Martingale convergence in the branching random walk, J. Appl. Probab. 14 (1977) $25-37$.

[7] J.D. Biggins, Uniform convergence of martingales in the one-dimensional branching random walk, in: Selected proceedings of the Sheffield Symposium on Applied Probability, 1989, in: I.V. Basawa, R.L. Taylor (Eds.), IMS Lecture Notes Monogr. Ser., vol. 18, 1991, pp. 159173.

[8] J.D. Biggins, Uniform convergence of martingales in the branching random walk, Ann. Probab. 20 (1992) $137-151$.

[9] J.D. Biggins, A.E. Kyprianou, Branching random walk: Seneta-Heyde norming, in: B. Chauvin, S. Cohen, A. Rouault (Eds.), Trees: Proceedings of a Workshop, Versailles, June 14-16, 1995, Birkhäuser, Basel, 1996.

[10] J.D. Biggins, A.E. Kyprianou, Measure change in multi-type branching, Advances of Applied Probability, in press.

[11] M. Bramson, Maximal displacement of branching Brownian motion, Comm. Pure Appl. Math. 31 (1978) 531-581.

[12] M. Bramson, Convergence of solutions to the Kolmogorov nonlinear diffusion equation to travelling waves, Mem. Amer. Math. Soc. 44 (1983) $1-190$.

[13] A. Champneys, S.C. Harris, J.F. Toland, J. Warren, D. Williams, Algebra, analysis and probability for a coupled system of reactiondiffusion equations, Philos. Trans. Roy. Soc. London (A) 350 (1995) 69-112.

[14] B. Chauvin, Multiplicative martingales and stopping lines for branching Brownian motion, Ann. Probab. 30 (1991) 1195-1205.

[15] B. Chauvin, A. Rouault, KPP equation and supercritical branching Brownian motion in the subcritical speed area. Application to spatial trees, Probab. Theory Related Fields 80 (1988) 299-314.

[16] B. Chauvin, A. Rouault, Supercritical branching Brownian motion and KPP equation in the critical speed area, Math. Nachr. 149 (1990) 41-59.

[17] B. Chauvin, A. Rouault, A. Wakolbinger, Growing conditioned trees, Stochastic Process. Appl. 39 (1991) 117-130.

[18] R. Durrett, Probability: Theory and Examples, Duxbury, Belmont, CA, 1996.

[19] E.B. Dynkin, Superprocesses and partial differential equations, Ann. Probab. 21 (1993) 1185-1262. 
[20] J. Engländer, A.E. Kyprianou, Local extinction versus local exponential growth for spatial branching processes, Ann. Probab., 2002, submitted for publication.

[21] A. Etheridge, An Introduction to Superprocesses, in: Univ. Lecture Ser., Amer. Math. Soc., Providence, RI, 2000.

[22] S. Evans, Two representations of a superprocess, Proc. Roy. Soc. Edinburgh Sect. A 125 (1993) 959-971.

[23] R.A. Fisher, The advance of advantageous genes, Ann. Eugenics 7 (1937) 355-369.

[24] S.C. Harris, D. Williams, Large-deviations and martingales for a typed branching diffusion: I, Astérisque 236 (1996) $133-154$.

[25] S.C. Harris, Convergence of a Gibbs-Boltzmann random measure for a typed branching diffusion, in: Séminaire de Probabilités, vol. XXXIV, 2000.

[26] S.C. Harris, Travelling-waves for the F-K-P-P equation via probabilistic arguments, Proc. Roy. Soc. Edinburgh Sect. A 129 (1999) 503517.

[27] P. Jagers, General branching processes as Markov fields, Stochastic Process. Appl. 32 (1989) 193-212.

[28] F.I. Karpelevich, M.Ya. Kelbert, Yu.M. Suhov, The branching diffusion, stochastic equations and travelling wave solutions to the equation of Kolmogorov-Petrovskii-Piskounov, in: N. Boccara, E. Goles, S. Martinez, P. Picco (Eds.), Cellular Automata and Cooperative Behaviour, Kluwer Academic, Dordrecht, 1993, pp. 343-366.

[29] M.Ya. Kelbert, Yu.M. Suhov, The Markov branching random walk and systems of reaction-diffusion (Kolmogorov-Petrovskii-Piskunov) equations, Comm. Math. Phys. 167 (1995) 607-634.

[30] J.F.C. Kingman, The first birth problem for an age-dependent branching processes, Ann. Probab. 3 (1975) $790-801$.

[31] A. Kolmogorov, I. Petrovskii, N. Piskounov, Étude de l'équation de la diffusion avec croissance de la quantité de la matière et son application a un problèm biologique, in: Moscow Univ. Math. Bull., vol. 1, 1937, pp. 1-25.

[32] T. Kurtz, R. Lyons, R. Pemantle, Y. Peres, A conceptual proof of the Kesten-Stigum theorem for multi-type branching processes, in: K.B. Athreya, P. Jagers (Eds.), Classical and Modern Branching Processes, in: Math. Appl., vol. 84, Springer-Verlag, New York, 1997, pp. 181-186.

[33] J.-F. LeGall, Spatial Branching Processes, Random Snakes and Partial Differential Equations, in: Lectures Math., Birkhäuser, Basel, 1999.

[34] O.D. Lyne, Travelling waves for a certain first-order coupled PDE system, Electron. J. Probab. 5 (2000), Paper 14.

[35] R. Lyons, A simple path to Biggins' martingale convergence theorem, in: K.B. Athreya, P. Jagers (Eds.), Classical and Modern Branching Processes, in: Math. Appl., vol. 84, Springer-Verlag, New York, 1997, pp. 217-222.

[36] R. Lyons, R. Pemantle, Y. Peres, Conceptual proofs of $L \log L$ criteria for mean behaviour of branching processes, Ann. Probab. 23 (1995) $1125-1138$

[37] H.P. McKean, Excursions of a non-singular diffusion, Z. Wahr. werv. Geb. 1 (1963) 230-239.

[38] H.P. McKean, Application of Brownian motion to the equation of Kolmogorov-Petrovskii-Piskunov, Comm. Pure Appl. Math. 29 (1975) $323-331$.

[39] J. Neveu, Multiplicative martingales for spatial branching processes, in: E. Çinlar, K.L. Chung, R.K. Getoor (Eds.), Seminar on Stochastic Processes, 1987, in: Progr. Probab. and Statist., vol. 15, Birkhaüser, Boston, 1988, pp. 223-241.

[40] P. Olofsson, The $x \log x$ condition for general branching processes, J. Appl. Probab. 35 (1998) 537-554.

[41] Pitman, One dimensional Brownian motion and three dimensional Bessel processes, Adv. Appl. Probab. 7 (1975) 511-526.

[42] T. Shiga, S. Watanabe, Bessel diffusions as a one-parameter family of diffusion processes, Z. Wahr. Verw. Geb. 27 (1973) $37-46$.

[43] A.V. Skorohod, Branching diffusion processes, Theory Probab. Appl. 9 (1964) $492-497$.

[44] K. Uchiyama, The behaviour of solutions of some non-linear diffusion equations for large time. 1, J. Math. Kyoto Univ. 18 (1978) 453-508.

[45] D. Williams, Path decomposition and continuity of local times for one-dimensional diffusions, Proc. London Math. Soc. 28 (1974) $737-$ 768. 